\title{
A Selective Impairment of Motion Perception Following Lesions of the Middle Temporal Visual Area (MT)
}

\author{
William T. Newsome and Edmond B. Paré \\ Department of Neurobiology and Behavior, State University of New York, Stony Brook, New York 11794
}

\begin{abstract}
Physiological experiments indicate that the middle temporal visual area (MT) of primates plays a prominent role in the cortical analysis of visual motion. We investigated the role of MT in visual perception by examining the effect of chemical lesions of MT on psychophysical thresholds. We trained rhesus monkeys on psychophysical tasks that enabled us to assess their sensitivity to motion and to contrast. For motion psychophysics, we employed a dynamic random dot display that permitted us to vary the intensity of a motion signal in the midst of masking motion noise. We measured the threshold intensity for which the monkey could successfully complete a direction discrimination. In the contrast task, we measured the threshold contrast for which the monkeys could successfully discriminate the orientation of stationary gratings. Injections of ibotenic acid into MT caused striking elevations in motion thresholds, but had little or no effect on contrast thresholds. The results indicate that neural activity in MT contributes selectively to the perception of motion.
\end{abstract}

Extrastriate visual cortex in primates comprises a mosaic of visual areas that can be distinguished on the basis of visual topography, anatomical connections, cortical architecture, and physiological response properties. A growing corpus of data indicates that several of these areas are specialized for the analysis of visual motion information. These areas appear to constitute a motion pathway that originates in striate cortex and terminates in higher cortical areas of the parietal lobe (reviewed by Maunsell and Newsome, 1987).

The salient physiological feature of this pathway is an elevated percentage of directionally selective neurons at each level. The pathway begins in layer $4 \mathrm{~B}$ of striate cortex, which is enriched in such cells relative to other striate laminae (Dow, 1974; Blasdel and Fitzpatrick, 1984; Livingstone and Hubel, 1984; Michael, 1985). Layer $4 \mathrm{~B}$ projects, in turn, to the middle temporal visual area $(\mathrm{MT})$, in which over $80 \%$ of the neurons are directionally selective (e.g., Dubner and Zeki, 1971; Maunsell and Van Essen, 1983a; Albright, 1984). MT receives additional inputs from striate cortex via indirect routes through V2 and V3

\footnotetext{
Received Aug. 6, 1987; accepted Nov. 19, 1987.

We are grateful to Dr. J. A. Movshon for providing the software used in generating the random dot displays, and to Dr. William Merigan for useful suggestions concerning the design and execution of the psychophysical cxpcriments. We also thank Drs. R. H. Wurtz and K. H. Britten for criticism of the manuscript, and Kate Melia and Sean Friend for technical assistance during the experiments. This work was supported by the National Eye Institute (R01-EY05603) and by a Sloan Research Fellowship to W.T.N.

Correspondence should be addressed to Dr. William T. Newsome at his present address: Department of Neurobiology, Stanford University School of Medicine, Stanford, CA 94305.
}

Copyright (C) 1988 Society for Neuroscience $0270-6474 / 88 / 062201-11 \$ 02.00 / 0$
(Maunsell and Van Essen, 1983c). These projections may also play a significant role in the motion pathway, since $40 \%$ of the neurons in V3 are directionally selective (Felleman and Van Essen, 1987).

Beyond MT, the motion pathway continues into the parietal lobe. MT projects directly to 2 cortical areas, the medial superior temporal area (MST) and the ventral intraparietal area (VIP), near the boundaries of Brodmann's area 7 (Maunsell and Van Essen, 1983c; Ungerleider and Desimone, 1986), and both of these areas appear to project to the inferior parietal lobule (see Van Essen, 1985). MST contains a high percentage of directionally selective neurons (Van Essen et al., 1981; Desimone and Ungerleider, 1986; Tanaka et al., 1986), but physiological recordings have yet to be made in VIP.

Until recently, the hypothesized role of this pathway in motion processing remained inferential, being based solely on electrophysiological data. However, behavioral experiments have now provided a firmcr basis for this hypothcsis. Newsomc ct al. (1985) showed that lesions of MT impair eye movements to moving visual targets while leaving eye movements to stationary targets unaffected. The impairment resulted from a visual deficit that appeared to be selective for moving stimuli. These experiments established a role for MT in the guidance of visual behavior, but yielded no information concerning the role of MT in perception.

In the present experiments, we examined the role of MT in perception by analyzing the effects of MT lesions on psychophysical thresholds. We trained rhesus monkeys on 2 tasks: a motion-sensitivity task involving a direction discrimination, and a contrast-sensitivity task involving an orientation discrimination. We found that MT lesions caused striking elevations of motion thresholds, while having little or no effect on contrast thresholds. MT thus appears to process information that is important for the perception of motion, as well as for the guidance of eye movements. A brief report of these results has appeared previously (Newsome and Paré, 1986).

\section{Materials and Methods}

We examined the effects of MT lesions in 3 hemispheres $(m 1, m 2$, and $w I)$ of 2 rhesus monkeys. Under surgical anesthesia and aseptic conditions, we implanted an eye coil (Judge et al., 1980), a head-holding device, and a stainless steel cylinder for microelectrode recording (Evarts, 1966,1968 ) in each animal. The eye coil was used in conjunction with a magnetic search coil apparatus to measure eye movements (Robinson, 1963; Fuchs and Robinson, 1966). As liquid rewards were employed in all behavioral paradigms, we controlled water intake so as to maintain each animal in a healthy, but motivated, state. The animals were comfortably seated in a primate chair during experiments and were returned to their home cages immediately following each session.

Visual stimuli. We employed a dynamic random dot display for psychophysical measurement of motion thresholds. The display was pre- 

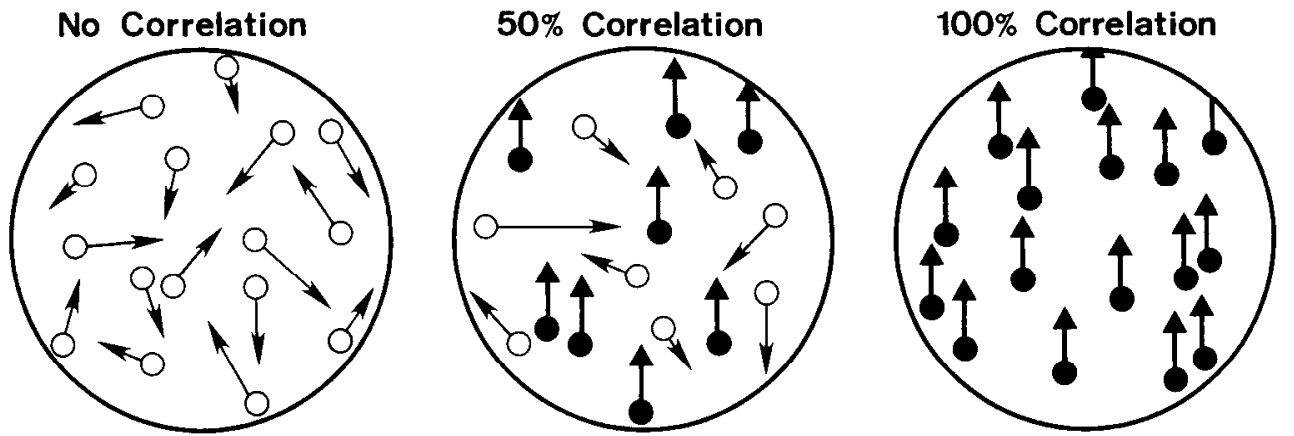

Figure 1. Schematic representation of the motion stimulus employed in the present study. A sequence of dots is plotted under computer control at random positions on a CRT screen. Each dot survives for a brief interval (20-30 $\mu \mathrm{sec})$, after which it disappears and is replaced by another randomly placed dot. The operator may specify that a certain percentage of the dots be replotted with a fixed spatial and temporal offset from their partner dots. This subset of dots constitutes a net motion signal that is embedded within a mask of random motion events. Left, The condition in which all dots are plotted randomly (no, or $0 \%$, correlation). There is no net motion in such a display, although there are many local motion events due to fortuitous pairings among the stream of randomly positioned dots (arrows). Right, The other extreme, in which all dots are replotted with a fixed spatiotemporal offset so that the motion of each dot is identical to that of the entire pattern (100\% correlation). Center, An intermediate display in which $50 \%$ of the dots comprise the "correlated" motion signal, while the other $50 \%$ are randomly plotted in a masking motion noise $(50 \%$ correlation $)$.

sented on a CRT screen under control of a PDP11/73 laboratory computer, using software provided by Dr. J. A. Movshon of New York University. In general, random dot displays have several virtues for use in motion psychophysics. Studying motion perception with traditional geometric stimuli is complicated by the fact that motion can be inferred from changes in position of recognizable features. Motion can be inferred in this manner even when primary motion-sensing mechanisms are inactive (consider, for example, the motion of the hands of a clock). Random dot displays, on the other hand, stimulate primary motionsensing mechanisms (e.g., directionally selective neurons), while minimizing familiar position cues (see Braddick, 1974; Morgan and Ward, 1980; Nakayama and Tyler, 1981; Williams and Sekuler, 1984). Random dot displays also permit other parameters, such as spatial and temporal frequency content, mean luminance, and contrast to be held constant across the entire visual display.

In addition to these general advantages, our display offers the particular advantage that the intensity of the motion signal can be specified precisely by varying the percentage of dots that actually carries the motion signal. In our basic display, dots are plotted in rapid succession at random locations on the CRT screen. Each dot survives for 20-30 $\mu \mathrm{sec}$ before it vanishes and is replaced by a new dot at a randomly selected screen location. When viewing this display, the overall impression is of twinkling visual noise in which many local motion events are present (by virtue of random pairings of successively plotted dots), but with no net motion in any direction. We refer to this as the "no correlation" or "zero percent" state (Fig. 1, left). $\Lambda$ t the other extreme is the " $100 \%$ correlation" state (Fig. 1, right). In this display each dot, upon vanishing, is replaced by a new dot with a fixed displacement in space and time $(d x$ and $d t)$ from its partner. The $100 \%$ correlation state corresponds to the traditional random dot pattern, in which the motion of each individual dot is identical to that of the whole pattern.

Our subjects, both monkey and human, usually viewed a stimulus that was intermediate between these 2 extremes. In the center panel of Figure 1, for example, $50 \%$ of the dots are plotted at random locations on the screen, while another $50 \%$ are in correlated motion. In this situation, a correlated motion signal is embedded in a masking motion noise, and the strength of the signal may be smoothly varied from zero to unity by changing the percentage of dots in corrrelated motion. The computer program gives the experimenter full control over this parameter, as well as over the spatiotemporal composition of the correlated motion events $(d x$ and $d t)$. The goal of our psychophysical measurements was to determine the threshold correlation for which the monkeys could reliably discriminate upward from downward motion (see below).

It is important to appreciate one subtlety of the algorithm by which the dots are generated. Each dot that appears on the screen has an equal chance of being selected to receive a correlated partner and thus contribute to the net motion signal. If, for example, the experimenter specifies a correlation value of $50 \%$ for a particular trial, each dot has a $50 \%$ chance of being succeeded by a correlated partner. The correlated partners, in turn, also have a $50 \%$ chance of being succeeded by yet another correlated partner. There exists, therefore, a $25 \%$ chance that a "streak" of 3 dots will occur, a $12.5 \%$ chance for a "streak" of 4 dots, etc. In the present study, all prelesion thresholds were below $10 \%$ correlation, and it was therefore rare for multidot streaks to appear in the display. This point is important because long-lived streaks might provide a local cue to which the animals could selectively attend. Since long-lived streaks are uncommon near our animals' thresholds, a monkey cannot gain an advantage by attending to selected local motion events since he has no way of distinguishing correlated from random events. Rather, the monkey must integrate information from the entire display to detect the correlated motion signal carried by pairs of dots that are spatially dispersed among the random motion events. This stimulus appears particularly appropriate for activating motion-sensing mechanisms in extrastriate visual areas such as MT. Small receptive fields, such as those in V1, can only sample a local region of the display, and therefore seem unlikely to detect the net motion signal at low correlations. Extrastriate neurons, however, have large receptive fields that may be suitable for encoding the net motion signal.

In many instances, it is of interest to know the density of dots in a random dot display, but this is not an easily addressable question in our case. Since dots are plotted singly at $150 \mu \mathrm{sec}$ intervals with a 20 $30 \mu \mathrm{sec}$ survival time, dot density in the display is usually 0 . However, because of the persistence of images in the visual system, an observer in fact perceives numerous dots at any instant in time. If one assumes a rough figure of $100 \mathrm{msec}$ for visual persistence, the dot density at a given point in time is about $1.7 \mathrm{dots} / \mathrm{deg}^{2}$. In our standard viewing aperture $\left(10^{\circ}\right.$ diameter), therefore, about 130 dots are visible at any point in time.

In the contrast-sensitivity task, we measured the threshold contrast for which the monkeys could successfully discriminate the orientation of a stationary sine wave grating (see below). Sine wave gratings of varying orientation, spatial frequency, and contrast were generated using a Picasso image synthesizer (Innisfree) under computer control. The contrast of a sine wave grating was calculated by the formula, $\left(L_{\max }-\right.$ $\left.L_{\min }\right) /\left(L_{\max }+L_{\min }\right)$, where $L_{\max }$ and $L_{\min }$ are the maximum and minimum luminances in the grating, as measured with a spot photometer.

Behavioral paradigms. Both motion and contrast thresholds were measured with 2-alternative, forced-choice psychophysical procedures. Figure $2 A$ illustrates the paradigm for measuring direction-discrimination thresholds. Each trial began with the appearance of a fixation target at one of 2 locations, FP1 or FP2. After the monkey foveated the target, the dot display appeared for $2 \mathrm{sec}$ within a circular aperture that was $10^{\circ}$ in diameler for experinents $m 1$ and $m 2$, and $12^{\circ}$ in diameter for experiment $w 1$. In all experiments the aperture was centered on the horizontal meridian, and the nearest edge of the aperture was $2^{\circ}$ from the fixation target. The net motion of the dot pattern was either upward or downward on a given trial, and was randomly varied between trials. Following the $2 \mathrm{sec}$ viewing interval, the fixation target was extinguished and 2 other targets appeared, one above the viewing aperture ("UP" target) and one below ("DOWN" target). The monkey indicated whether 
the net motion of the dots was upward or downward by making a saccadic eye movement to the corresponding target. Correct choices were rewarded with a drop of water; incorrect choices were punished with a short time-out interval. If the monkey broke fixation before the $2 \mathrm{sec}$ viewing interval was completed, the trial was aborted and the data discarded. By limiting premature guessing in this manner, we hoped to ensure that the monkeys made a "mature" judgement on each trial.

Psychophysical data were obtained in blocks of trials in which the percentage of dots in correlated motion was varied using a staircase procedure. The correlation percentage was set well above threshold for the initial trial. When the monkey completed a trial correctly, there was a $37 \%$ chance of a step to a weaker motion signal (lower correlation) on the following trial. When the monkey chose incorrectly, the motion signal always underwent a step increase in intensity (higher correlation) on the following trial. This ratio of step changes for correct versus incorrect behavior ensured that the animal would be rewarded on 60$70 \%$ of the trials in a block. The steps in correlation were equally spaced on a logarithmic scale.

Typically, the monkey would complete several trials correctly at the beginning of a block and the correlation level would fall quickly toward threshold. The monkey's behavior would then oscillate about threshold for the duration of the block of trials. Each block was 80 trials long and constituted a single threshold measurement. If the monkey responded incorrectly on 3 consecutive trials for motion in a particular direction, correction trials were presented until the monkey responded correctly to that direction of motion. This procedure ensured that the animal did not develop a bias for one saccade target over the other. The correlation level did not change on correction trials, and the correction trials did not count toward the 80-trial threshold determination.

As stated above, our software enabled us to specify the spatiotemporal composition ( $d x$ and $d t)$ of the correlated motion signal. In this study we measured motion thresholds for 5 different spatial intervals $(d x)$, ranging on a logarithmic scale from $0.04^{\circ}$ to $0.64^{\circ}$. The temporal interval $(d t)$ was $45 \mathrm{msec}$ for all threshold measurements. This range of spatial intervals was selected during preliminary experiments so as to include the region of lowest psychophysical thresholds.

For each spatiotemporal condition tested (combination of $d x$ and $d t$ ), we measured thresholds simultaneously in both the left and right visual hemifields. We accomplished this by alternating the fixation point randomly from trial to trial between locations $\Gamma P 1$ and FP2 (Fig. 2A). However, the staircase logic in one hemifield was unaffected by the monkey's performance in the other hemifield; the stimuli were temporally interleaved between the 2 hemifields, but threshold measurements were independent of each other. Since the excitatory receptive fields of MT neurons are substantially restricted to the contralateral hemifield, this procedure provided an excellent intra-animal control for extraneous behavioral variables, such as attention, water satiation, etc.

Contrast thresholds were measured using similar methods, as illustrated in Figure $2 B$. The monkey fixated the same targets and viewed the stimuli through the same circular aperture used for measurement of motion thresholds. In this case, however, the stimuli were stationary sine wave gratings, and our procedure was designed to measure the threshold contrast for which monkeys could successfully discriminate the orientation of the grating. For a particular trial, the grating was oriented either horizontally or vertically, and the monkey again indicated his choice by making a saccadic eye movement to the corresponding target ("HORIZONTAL" target or "VERTICAL" target in Fig. 2B). The contrast of the grating was varied from trial to trial by the same rules described above for motion correlation. Again, single threshold measurements were made in 80-trial blocks. For one experiment $(\mathrm{ml})$, we measured contrast thresholds simultaneously in the 2 hemifields, as described for the motion thresholds. For experiment $m 2$ (the first, chronologically, to be performed), contrast thresholds were not available since the monkey had not learned this task at the time of the lesion. The second monkey (experiment $w 1$ ) had difficulty executing the orientation discrimination with trials interleaved between the 2 hemifields, and we found it necessary to restrict stimuli to a single hemifield in order to obtain consistent threshold values. The same monkey, however, had no trouble performing the motion task with interleaved trials, and we therefore obtained motion thresholds for both hemifields. We believe this peculiarity may have been related to the different placement of saccade targets for the 2 tasks (up and down versus up and left; see Fig. 2).

It is worth commenting on our choice of a saccadic eye movement as an operant response rather than the more widely used bar press or
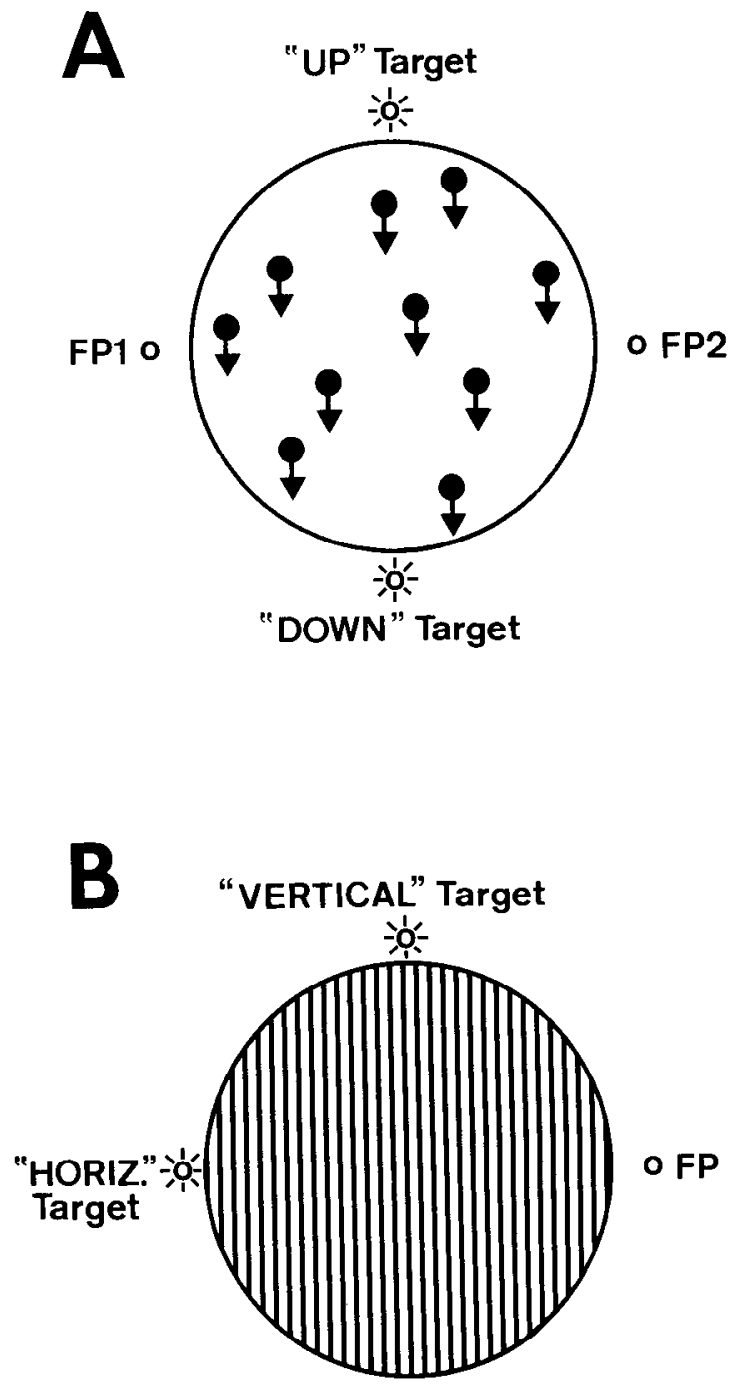

Figure 2. Behavioral paradigms employed for measuring psychophysical thresholds. $A$, For motion thresholds, the monkey viewed a dynamic random dot pattern that appeared within a circular aperture. Net motion of the dot pattern was either upward or downward for a given trial. The monkey fixated a point of light at location FP1 or FP2 while viewing the dot pattern perifoveally for $2 \mathrm{sec}$. At the end of the viewing interval, the fixation point vanished and 2 saccade targets appeared, one above the viewing aperture and the other below. The monkey was required to make a saccade to the target that corresponded to the direction of motion of the dot pattern ("UP" or "DOWN" target). A correct choice was rewarded with a drop of water. The intensity of the motion signal (percentage correlation) was varied from trial to trial in order to measure the threshold intensity for which the monkey could successfully discriminate the direction of motion. The fixation point was randomly alternated between locations FP1 and FP2 so that thresholds could be measured simultaneously in each hemifield (see text). $B$, For contrast thresholds, the monkey viewed a stationary sine wave grating through the same aperture employed during measurement of motion thresholds. Again, the monkey was required to fixate a small target (e.g., FP) while viewing the stimulus aperture perifoveally. Following the $2 \mathrm{sec}$ viewing period, the monkey indicated whether the grating was oriented vertically or horizontally by making a saccade to the corresponding target ("VERTICAL" or "HORIZONTAL" target). Grating contrast was varied from trial to trial in order to determine the threshold contrast for which the monkey could successfully discriminate the orientation of the grating. 


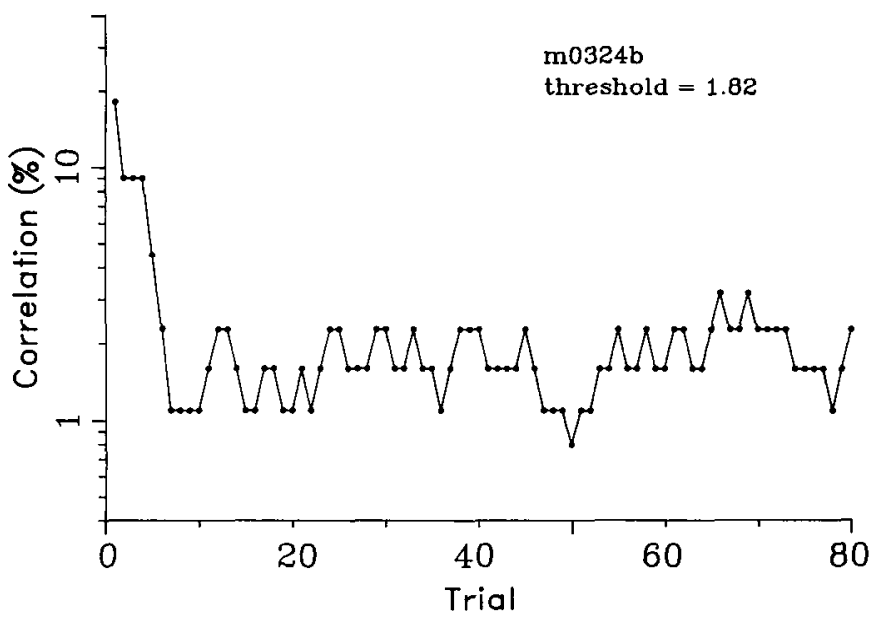

Figure 3. An example of a threshold determination for the motionsensitivity task. The ordinate shows the percentage of dots in correlated motion for each trial of an 80-trial staircase. The correlation value was well above threshold on the initial trial, and the probability of a decrease in correlation was $37 \%$ for each correct response. The correlation value increased following each incorrect response. Until the monkey's first incorrect response, the steps in correlation value were double the normal size. This allowed the monkey to reach threshold values in fewer trials. We obtained a quantitative measure of the threshold from the last 60 trials in each block, a period in which the monkey's behavior oscillated about threshold. The threshold was calculated as the mean of the correlation values at each turning point (maxima and minima) during the last 60 trials. In this example, the threshold was $1.82 \%$.

lever release. It seemed to us that the eye movement response would be more quickly learned, since exploration of the visual environment with eye movements is a natural and frequent component of a monkey's behavioral repertoire. We also felt confident that MT lesions would have no effect on the operant response itself, since a previous study showed that these lesions have no effect on eye movements to stationary targets (Newsome et al., 1985). Our expectations seemed justified during training. After learning a standard saccade task, the monkeys learned to choose between saccade targets on the basis of the motion signal in $2-3 \mathrm{~d}$. The bulk of the training time (2-3 months for each task) was consumed waiting for prelesion thresholds to settle to asymptotic values.

Threshold calculation. Figure 3 illustrates the correlation value reached on each trial during a single 80-trial threshold measurement. The monkey reached threshold values within 10 trials and then oscillated about threshold for the duration of the block. We obtained a quantitative measurement of the threshold by averaging the correlation values at the "turning points" (maxima and minima) during the period of oscillatory behavior (last 60 trials). If an odd number of turning points occurred during the last 60 trials, the final turning point was excluded from the calculation, so that equal numbers of maxima and minima were used. Threshold values calculated in this manner are similar to those obtained with other techniques, such as probit analysis, and have the advantage of being computationally simpler (Levitt, 1971). In the example shown in Figure 3, the threshold value obtained was $1.8 \%$, indicating that the monkey could discriminate upward from downward motion when as few as $1.8 \%$ of the dots were in correlated motion.

Lesions. The lesions were made by injecting small volumes of the neurotoxin, ibotenic acid, into MT. Neurotoxins such as ibotenic acid have the useful property of selectively killing cell bodies while leaving fibers of passage in the underlying white matter unharmed (see Olney, 1983). The techniques for these injections have been described in detail previously (Newsome et al., 1985; Dursteler et al., 1987). Briefly, we mapped MT with microelectrodes, and then implanted a stainless steel guide tube that was positioned about $3 \mathrm{~mm}$ above the target region of MT. The target region was selected to correspond topographically to the region of visual space in which the stimulus aperture was located. After implanting the guide tube, we confirmed its location by recording with a microelectrode inserted into MT through the guide tube. Using the microdrive scale, we determined the depth of MT below the end of the guide tube, and then inserted a Hamilton syringe needle through the guide tube so that its tip rested at the target depth. We then injected

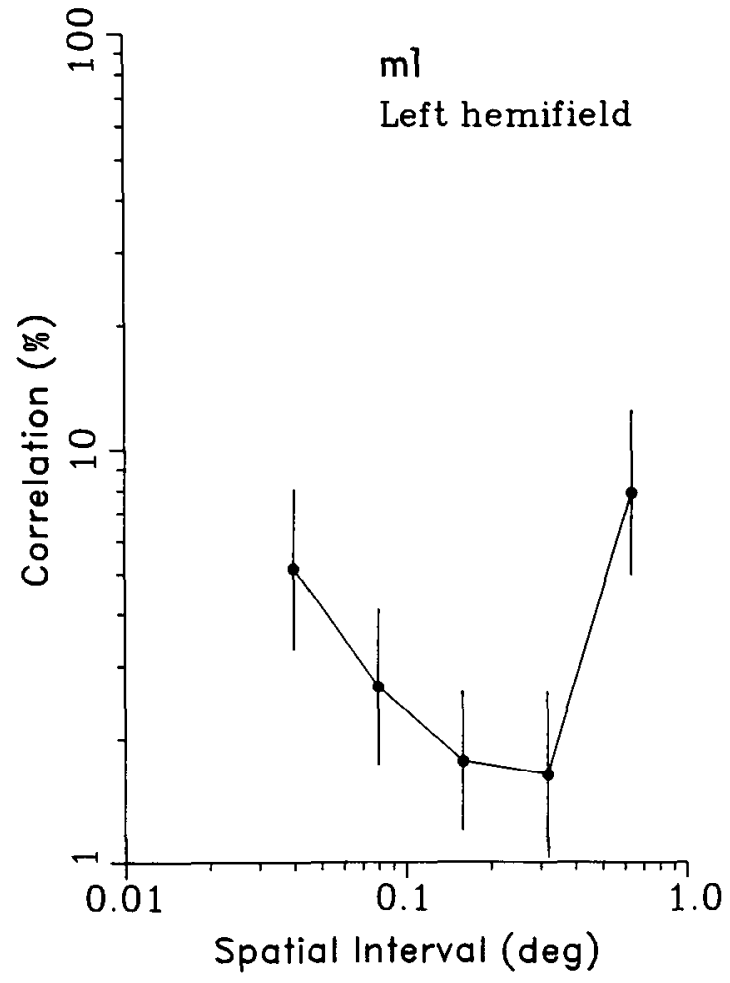

Figure 4. Normal psychophysical performance exhibited by the monkey in experiment $m l$. We measured thresholds at 5 different spatial intervals, ranging from $0.04^{\circ}$ to $0.64^{\circ}$. The temporal interval was constant at $45 \mathrm{msec}$. The monkey viewed the dot display perifoveally through a circular aperture $10^{\circ}$ in diameter. The aperture was centered on the horizontal meridian, and its nearest edge was $2^{\circ}$ from the fixation point. Solid circles show the mean value of 10 or more threshold determinations for each spatial interval; error bars, standard deviation of those measurements. The monkey was remarkably sensitive to the motion signal, being able to discriminate the direction of motion for correlation values as low as $2 \%$ at the best spatial intervals.

$2 \mu \mathrm{l}$ of ibotenic acid at a rate of $0.2 \mu \mathrm{l} / \mathrm{min}$. After completing the injection, we withdrew the syringe needle $2 \mathrm{~mm}$, allowed it to sit for 10 min, then withdrew it the rest of the way. Psychophysical testing resumed the day after the injection was made.

Histology. Following perfusion with $10 \%$ formalin, the brain was equilibrated in a $30 \%$ sucrose solution and sectioned at $50 \mu \mathrm{m}$ intervals. Every tenth section was stained for cell bodies with cresyl violet, and adjacent sections were stained for myelinated fibers by the method of Gallyas (1979). Lesions were identified and their boundaries plotted from cresyl violet-stained sections; the boundaries of MT were located in the myelin-stained sections (see Allman and Kaas, 1971; Van Essen et al., 1981). For a given hemisphere, the full extent of the lesion and the architectonically defined borders of MT were then displayed on a 2-dimensional map of the cortex constructed by the method of Van Essen and Maunsell (1980). These "flattened" maps of the cortex permit quick visualization of the lesions and their location with respect to the boundaries of the target visual area (see Fig. 10).

\section{Results}

The data described here are from lesions of MT in 3 hemispheres in 2 monkeys. For 2 of the hemispheres ( $m l$ and $w 1)$, we obtained psychophysical thresholds for both the motion and the contrast task, as described in Materials and Methods. The basic pattern of results is apparent in both of these experiments, and we will present these data first. We will then present results for the third hemisphere, $m 2$, in which we made a longitudinal series of lesions to investigate the mechanisms underlying recovery from the perceptual deficits. 

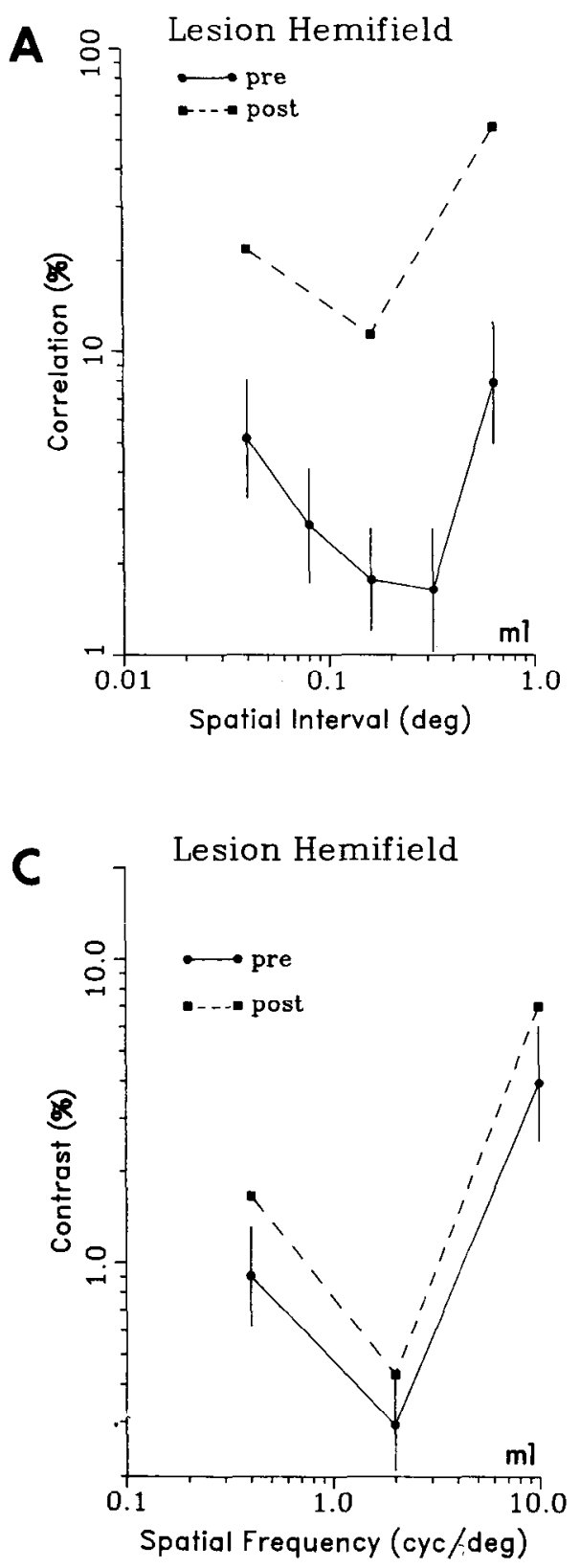
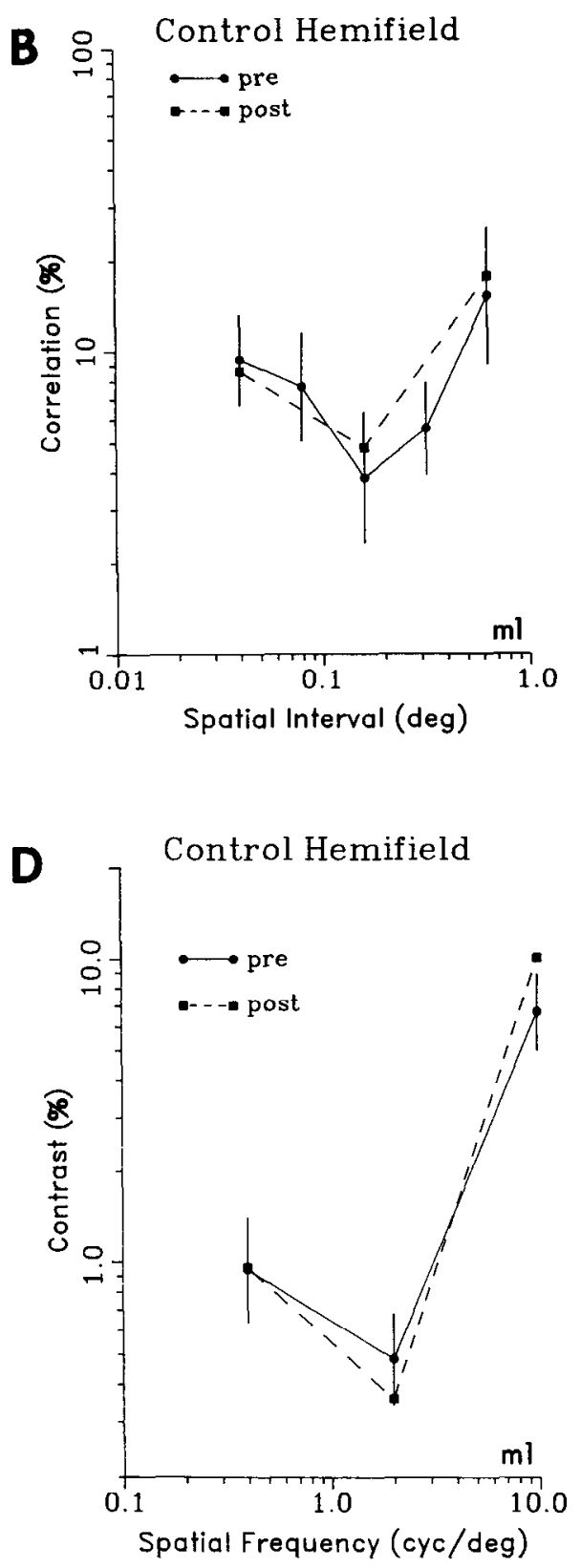

Figure 5. The psychophysical effects of an ibotenic acid injection into MT in experiment $m 1 . A-D$, Solid line and error bars indicate the mean prelesion threshold and standard deviation for each condition tested; dashed line, postlesion thresholds obtained $24 \mathrm{hr}$ after the MT injection. Error bars are not shown for the postlesion data, since each point represents a single threshold measurement for that spatial interval. $A$, Motion thresholds for 5 different spatial intervals in the test (contralateral) hemifield. The injection caused a 4-8fold increase in motion thresholds in this hemifield. $B$, Motion thresholds in the control (ipsilateral) hemifield. Since thresholds remained normal in this hemifield, the deficits in the test hemifield cannot be attributed to anomalies in the animal's level of water satiation, attention, etc. Prelesion thresholds in the control hemifield were higher than those in the test hemifield because the control hemisphere had already been subject to a complete unilateral lesion of MT in experiment $m 2$ (see Recovery of function, in Results). The previous experiment $(m 2)$ has no bearing on the interpretation of the present experiment, since the control data are only intended to show that the animal's overall motivational state was normal. $C$, Contrast thresholds for 3 different spatial frequencies in the test hemifield. The injection appears to have caused a slight elevation of contrast thresholds in the test hemifield, but the effect is far smaller than that shown in $A$ for the motion thresholds. $D$, Contrast thresholds in the control hemifield. The injection had no effect on contrast thresholds in this hemifield.

\section{The motion task: normal psychophysics}

In the motion-sensitivity task, we measured the threshold percentage of correlated dots for which the monkey could discriminate upward from downward motion (see Materials and Methods). Figure 4 illustrates normal prelesion performance for one monkey. Each data point represents the mean of 10 or more threshold measurements for a particular spatial interval, and the error bars indicate the standard deviation of the threshold values. The monkey was remarkably sensitive to the motion signal: for the best spatial intervals, he discriminated upward from downward motion when as few as $2 \%$ of the dots were in correlated motion. This performance compares favorably to the best thresholds we have observed in human subjects (W. T. Newsome, S. Friend, and K. Melia, unpublished observations). Over the range of spatial intervals tested, the data conformed to a U-shaped curve, with maximum sensitivity between $0.1^{\circ}$ and $0.4^{\circ}$. Performance deteriorated rapidly for spatial intervals greater than $0.64^{\circ}$ or less than $0.04^{\circ}$. These spatial limits correspond well to the values reported for the "short-range" motion mechanism at similar retinal eccentricities (Baker and Braddick, 1985). This similarity suggests that the neural mechanisms investigated in the present study may be common to a broad range of perceptual effects ascribed to the "short-range" mechanism (see Anstis, 1980; Braddick, 1980; Nakayama, 1985).

\section{Selectivity of the perceptual deficits}

Figure 5 illustrates the effect of a chemical lesion of MT on psychophysical thresholds in experiment $m l$. The lesion was unilateral and largely restricted to MT (see Fig. 10A). Figure $5 A$ depicts motion thresholds in the affected (contralateral) hemifield, while Figure $5 B$ shows motion thresholds in the control (ipsilateral) hemifield. The solid lines and error bars indicate prelesion performance, and the dashed lines illustrate psycho- 

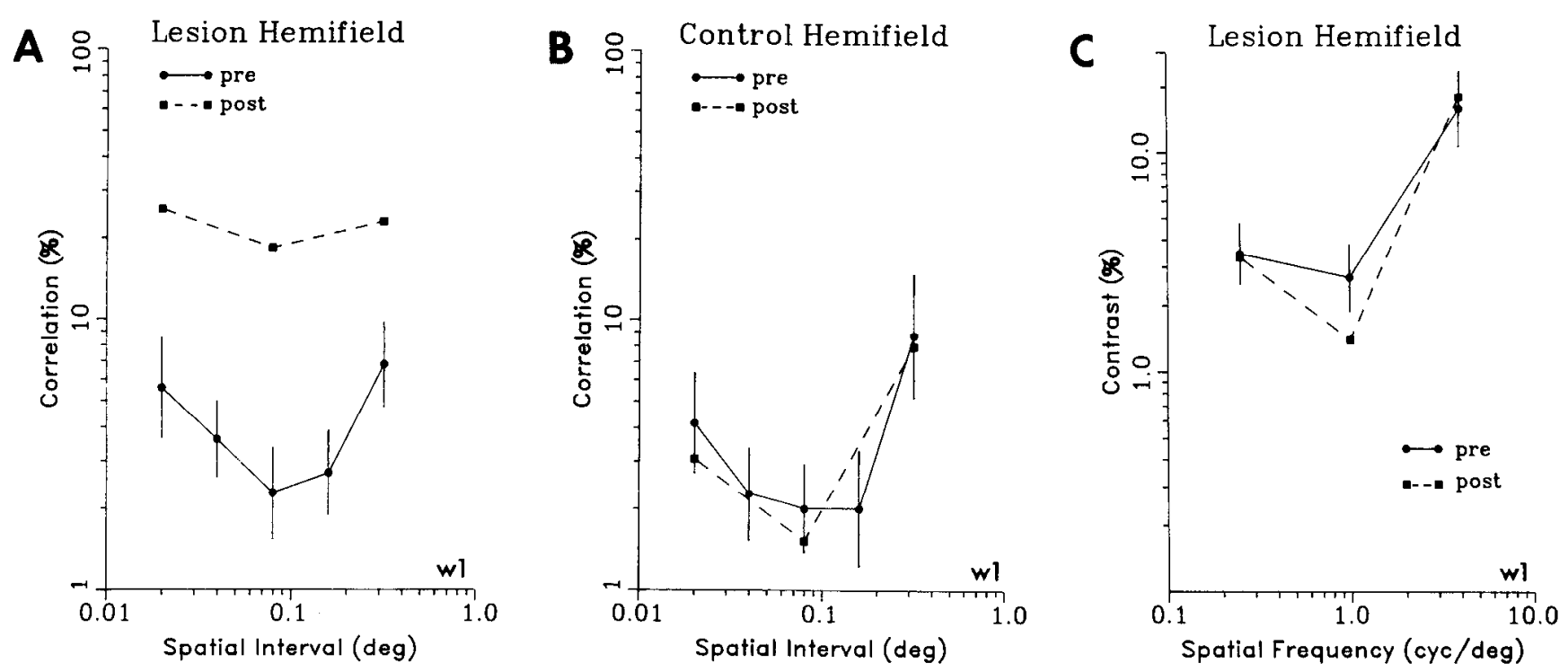

Figure 6. The psychophysical effects of an ibotenic acid injection into MT in experiment $w 1$. Solid line and error bars in $A-D$ indicate the mean prelesion threshold and standard deviation for cach condition testcd; dashed line, postlesion thresholds obtained $24 \mathrm{hr}$ after the MT injection. $A$, Motion thresholds for 5 different spatial intervals in the test (contralateral) hemifield. Again, the MT lesion caused striking elevations of motion thresholds in the test hemifield. $B$, Motion thresholds were within the normal range in the control (ipsilateral) hemifield. $C$, The MT injection had no effect on contrast thresholds in the test hemifield.

physical thresholds obtained $24 \mathrm{hr}$ after the injection of ibotenic acid into MT. The lesion caused dramatic threshold elevations for each of the 3 spatial intervals tested in the contralateral hemifield: thresholds increased by $400-800 \%$ (Fig. $5 A$ ). In con-

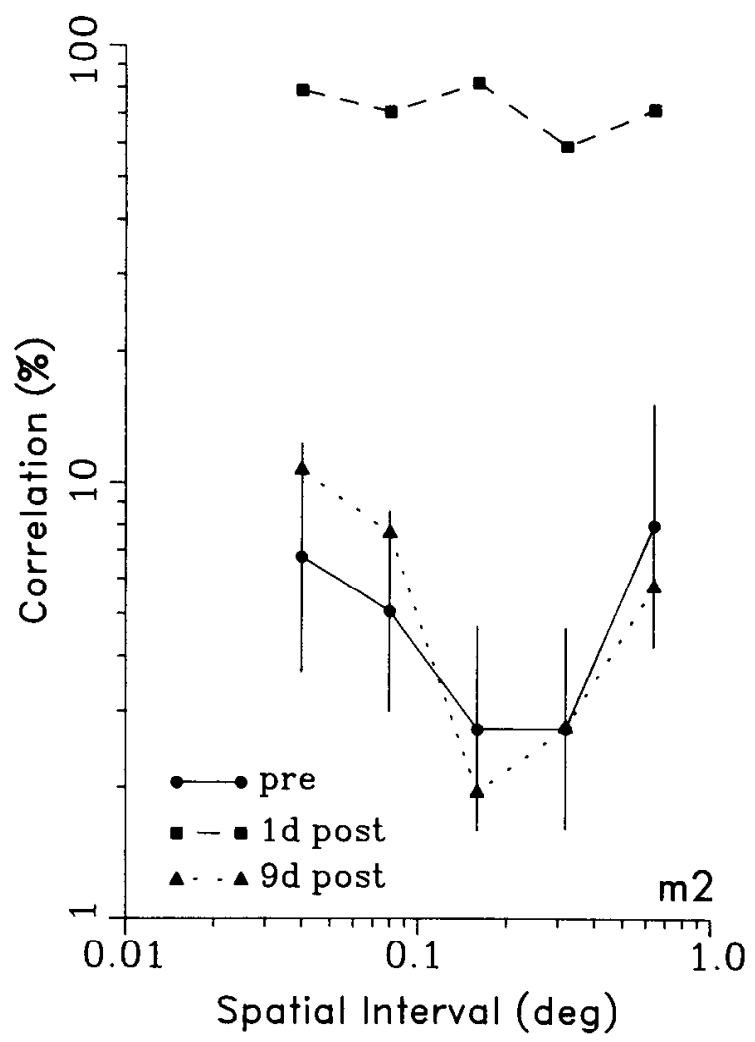

Figure 7. Recovery of function following 2 injections of ibotenic acid into MT in experiment $m 2$. Solid line and error bars indicate mean prelesion thresholds and standard deviations; dashed line, elevation of motion thresholds $24 \mathrm{hr}$ after the MT injections. The dotted line shows that the monkey's thresholds returned to normal levels by $9 \mathrm{~d}$ postlesion. trast, thresholds measured simultaneously in the control hemifield were entirely normal; Figure $5 B$ shows that all 3 thresholds from the control hemifield were within the range of prelesion performance. The fact that the monkey's behavior was unaffected in the control hemifield indicates that the impairment in the test hemifield resulted from the MT lesion and not from extraneous behavioral factors, such as water satiation or variation in the animal's level of attention.

Figure $5, C, D$, shows the effects of the MT lesion on the contrast-sensitivity task. Again, the solid lines indicate prelesion performance while the dashed lines depict thresholds measured $24 \mathrm{hr}$ after the injection of ibotenic acid into MT. We measured contrast thresholds at 3 spatial frequencies that spanned the most sensitive range for this retinal eccentricity. This monkey was extremely sensitive to contrast, performing better on this task than a human subject tested for comparison. Figure $5 C$ shows that postlesion thresholds were slightly above the mean prelesion level at all 3 spatial frequencies in the test hemifield. It is possible that these small increases represent a genuine effect of the MT lesion, but it is clear that the effect is far less than that for the motion thresholds illustrated in Figure $5 \mathrm{~A}$. As expected, contrast thresholds in the control hemifield were completely unaffected by the MT lesion (Fig. $5 D$ ).

We obtained the same pattern of results in experiment $w 1$, illustrated in Figure 6. Prelesion performance on the motion task (solid lines, Fig. 6, $A, B$ ) was similar to that of the first monkey and of human subjects. Figure $6 \mathrm{~A}$ depicts motion thresholds obtained in the test hemifield $24 \mathrm{hr}$ after an injection of ibotenic acid into MT. Again, the MT lesion caused striking threshold elevations in the test hemifield, while motion thresholds in the control hemifield appeared completely normal (Fig. $6 B)$.

Figure $6 C$ shows the monkey's performance on the contrastsensitivity task in the test hemifield. This monkey's prelesion thresholds were considerably higher than those for the first animal, but we are convinced that this represents the best the monkey could do, since 2 months of practice produced no fur- 
A

\section{STS - Posterior bank}

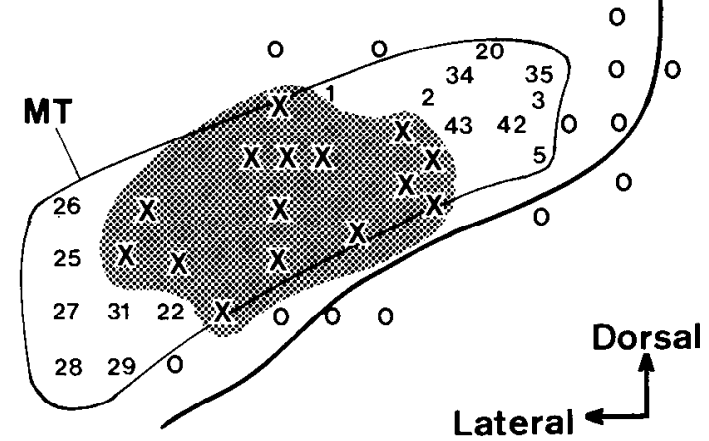

B

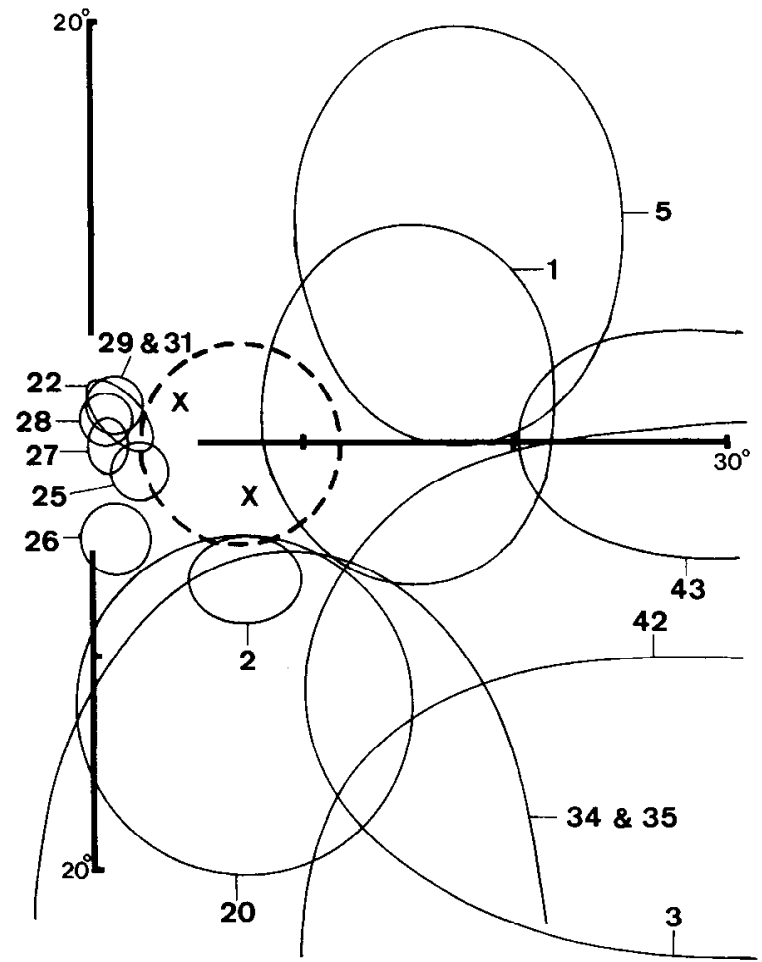

Figure 8. An electrophysiological map of MI generated in hemisphere $m 2$ subsequent to the behavioral recovery illustrated in Figure 7. A, Reconstruction of the posterior bank of the superior temporal sulcus (STS) based on electrophysiological recording. We identified surviving regions of MT by the characteristic direction selectivity of its neurons, by strength of the visual responses, by visual topography, and by comparison with a prelesion map of MT. The boundary of MT is outlined with a solid black line, and a heavy-gauge line shows the medial and ventral boundaries of the STS. The numbered recording sites illustrate penetrations in which we encountered healthy cortex in MT; $\times$ 's, penetrations that passed through the area of the lesion; $O$ 's, penetrations that missed MT. The shaded area shows the total extent of the lesion, which covered about a third of MT. We observed no spontaneous neural activity and could isolate no neurons within the area of the lesion. This map is a planar projection of physiological recordings trom a structure that is buried in a major sulcus, and scale is not, therefore, strictly accurate. However, a general notion of scale can be obtained, since the electrode penetrations at sites 27-29 and 31 form a square grid $1 \mathrm{~mm}$ on each side. $B$, Multiunit receptive fields mapped at recording sites in MT. Receptive fields are numbered according to their corresponding ther improvement in performance. The postlesion thresholds in Figure $6 C$ demonstrate that the MT lesion had no effect whatsoever on contrast thresholds in this experiment. Together, the results of experiments $m l$ and $w l$ indicate that MT lesions impair visual perception, and that the impairment is selective for the perception of motion.

\section{Recovery of function}

Figure 7 documents the recovery of function that followed the initial ibotenic acid injections in experiment $m 2$. The solid line and error bars indicate the mean prelesion thresholds with their standard deviations. This hemisphere received two $1 \mu \mathrm{l}$ injections of ibotenic acid into MT, and the dashed line in Figure 7 shows that the animal was profoundly impaired $24 \mathrm{hr}$ after the injections. The monkey had difficulty with the discrimination for correlation valucs as high as $80-100 \%$. However, with daily practice, the monkey recovered normal perceptual thresholds by $9 \mathrm{~d}$ postlesion (dotted line, Fig. 7). The time course of recovery varied from 3-4 d for small lesions, such as those in experiments $m 1$ and $w 1$, to 1-2 weeks for larger lesions, such as that in experiment $m 2$. The time course of recovery was similar to that previously reported for eye movement deficits that follow MT lesions (Newsome et al., 1985).

There are at least 3 classes of mechanism that may play a role in the recovery process. First, since the MT lesions created by small injections of ibotenic acid are subtotal, surviving cells within MT may have receptive fields that overlap the region of the visual field in which the stimuli are presented. Such cells may, after an adapting period, be able to assume the functions of the lost tissue. Secondly, plastic changes may occur in the representation of visual space in MT. Merzenich and Kaas and thcir colleagues have shown that plastic changes occur in the representation of the body surface in somatosensory cortex following peripheral lesions (Merzenich et al., 1983a; b, 1984). If similar changes occur near lesion boundaries in MT, the newly expanded receptive fields of these cells might play a role in behavioral recovery. Finally, recovery could be mediated by parallel pathways involving other visual areas.

In order to distinguish among these possibilities, we performed a series of electrophysiological recordings and additional lesions in hemisphere $m 2$. Following the recovery illustrated in Figure 7, we remapped MT with microelectrodes to determine (1) whether there were any obvious changes in topography that might account for the recovery, and (2) whether the surviving receptive fields overlapped the region of visual space in which we presented our stimuli. Figure $8 \mathrm{~A}$ shows a schematic outline of MT as reconstructed from the electrophysiological recordings. $\Lambda$ solid black line indicates the boundaries of MT, and a second line depicts the medial and ventral extent of the superior temporal sulcus (STS). The various numbers and symbols show physiological recording sites as reconstructed on the basis of microdrive depth and location within the recording cylinder. Numbered recording sites are those in which the electrode encountered healthy cortex in MT. The $\times$ 's represent recording sites within the area of the lesion. We encountered no cells in the latter penetrations; the only audible neural activity was an

recording sites in $A$. Dashed circle indicates the region of visual space occupied by the stimulus aperture in this experiment; $X$ 's show the topographic locations of the 2 ibotenic acid injections. 


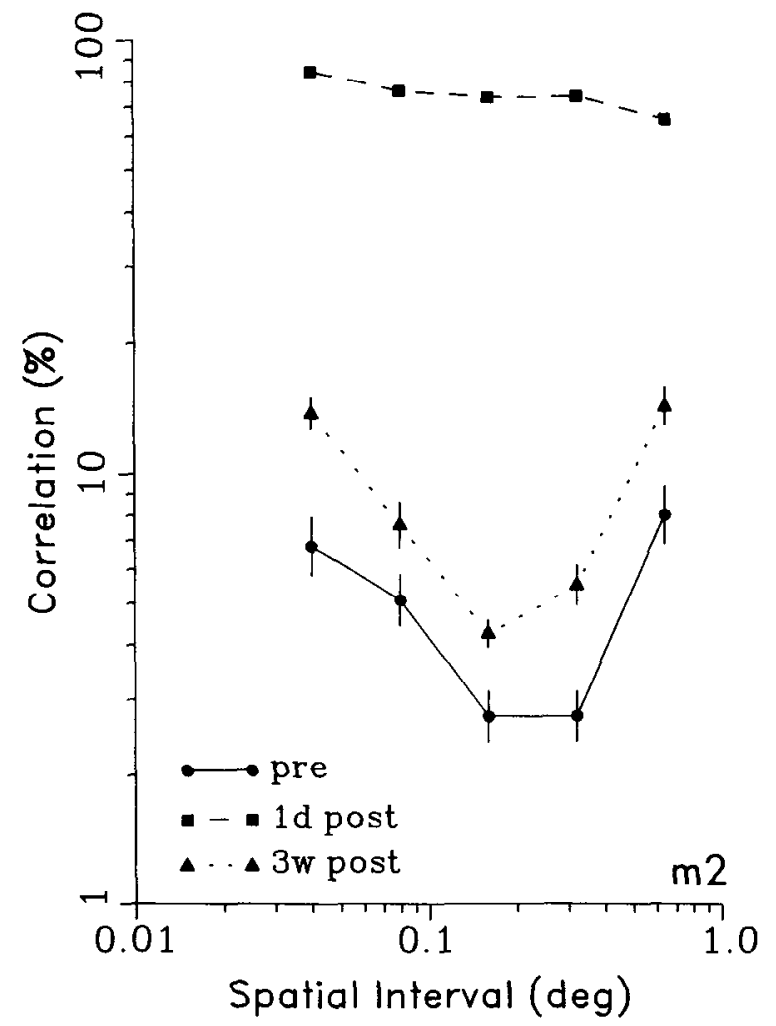

Figure 9. Recovery of function following a complete unilateral lesion of MT in experiment $m 2$. Solid line indicates prelesion thresholds for this experiment; dashed line, postlesion thresholds obtained $24 \mathrm{hr}$ after the second round of injections in this hemisphere; dotted line, postlesion thresholds 2-3 weeks postlesion. Symbols for the prelcsion and the 3 week postlesion data represent the mean value of at least 10 threshold measurements for each condition; error bars, standard error of the mean. We tested this animal until 5 months postlesion, but observed little recovery beyond that evident at 3 weeks. The residual deficit thus appeared to be permanent.

occasional faint "swish" response that may have signified the presence of functional afferents. The shaded region indicates the extent of the lesion, as deduced from these penetrations. The $O$ 's represent recording sites in healthy cortex outside of MT, or penetrations that missed the STS altogether. In all of these recordings, we identified $\mathrm{MT}$ by the characteristic direction selectivity of its neurons, by the strength of the response, by visual topography, and by comparison with the map of MT obtained during prelesion recordings.

As shown in Figure $8 A$, the 2 injections of ibotenic acid destroyed about a third of MT, leaving a patch of healthy cortex near the foveal representation (lateral and ventral) and another patch of healthy cortex in the representation of the far periphery (dorsal and medial). Figure $8 B$ shows the multiunit receptive field mapped at each of the numbered recording sites in Figure $8 A$. Also depicted in Figure $8 B$ is the location of the circular aperture through which the monkey viewed the visual stimuli (dashed line) and the topographic locations of the 2 injections of ibotenic acid ( $x$ 's). As expected, recording sites 22-31 yielded a cluster of receptive fields near the center of gaze, while the other recording sites yielded peripheral receptive fields.

Two conclusions are reasonably clear from the data in Figure 8. First, a few receptive fields overlap the region of visual space in which our stimuli appeared ( 1 and 25 , for example). It is conceivable that such receptive fields might transmit sufficient information from this region of visual space to play a role in the recovery process. Second, our recordings produced no clear evidence for plastic changes that might account for the behavioral recovery. Although the somewhat coarse scale of our recordings may have precluded detection of subtle changes in topography or receptive-field size, it seems unlikely that such changes were of sufficient magnitude to "fill in" the entire region of visual space affected by the lesion: a portion of the representation of visual space in MT appeared to be missing entirely following the lesion. Quantitative measurements of receptivefield size showed that the multiunit receptive fields in Figure 8 were within the range normally observed in MT (see Desimone and Ungerleider, 1986).

Since some MT receptive fields did overlap the stimulus aperture following behavioral recovery in experiment $m 2$, we made another series of ibotenic acid injections in this hemisphere, with the goal of creating a complete unilateral lesion of MT. We made 3 injections ( $2 \mu \mathrm{l} \mathrm{each}$ ) in the foveal representation of MT and an additional 3 injections in the peripheral representation. Histological reconstruction indicated that the lesion included all of MT in this hemisphere (see Fig. 10B). Figure 9 depicts the monkey's performance following the second round of injections. The solid line and error bars show the animal's prelesion performance, while the dashed line represents thresholds obtained $24 \mathrm{hr}$ after the injections. The deficit was fully reinstated following the injections, with thresholds for each spatial interval falling near the maximum correlation of $100 \%$. After 3 weeks of practice, however, the monkey had again recovered to the extent shown shown by the dotted line in Figure 9. We monitored the animal's performance until 5 months postlesion, and we observed little recovery beyond the level reached after 3 weeks. The chronic deficit signified by the dotted line in Figure 9 appeared, therefore, to be permanent. These results indicate that complete MT lesions can result in permanent perceptual deficits, but it is also clear that considerable recovery is possible in the absence of MT. This recovery presumably depends on motion information transmitted via pathways in parallel with those through MT.

\section{Histological reconstruction}

We used histological sections stained for cell bodies and myelinated fibers to reconstruct the lesions from experiments $m 1$ and $m 2$. The monkey in which we performed experiment $w 1$ is still alive and is being used in a related study. The lesion in experiment $w l$ was made by identical techniques and with similar results as the lesion in experiment $m l$. It seems likely, therefore, that the reconstruction for experiment $m l$ is indicative of the cortical damage in experiment $w 1$ as well.

We displayed the reconstructions on flattened maps of the cortex, as illustrated in Figure 10. Figure $10 \mathrm{~A}$ shows the partial lesion of $\mathrm{MT}$ that resulted from the restricted ibotenic acid injection in experiment $m 1$. MT is outlined on the posterior bank of the STS, and the extent of the lesion is shown by the solid black and stippled regions. The solid black region depicts cortex in which the lesion extended through the entire 6 layers, and the stippled region indicates cortex in which damage was restricted to a subset of the layers. The bulk of the damage occurred near the injection site in MT. Slight damage was visible in the upper layers of cortex in a location on the anterior bank of the STS that was directly apposed to the injection site on the posterior bank. 

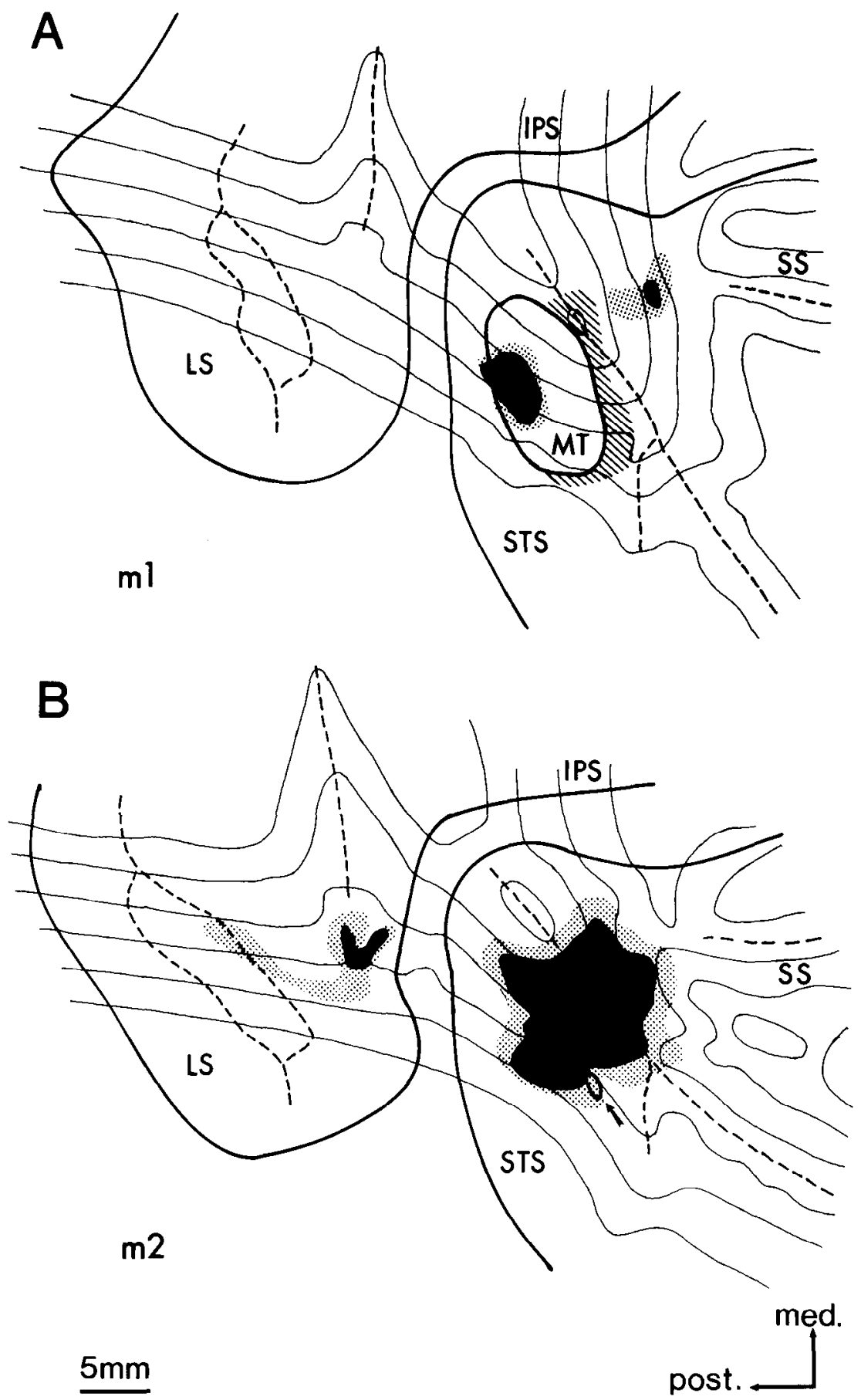

Figure 10. Histological reconstruction of the lesions in experiments $m l$ and $m 2$. The lesion boundaries were identified in cresyl violet-stained sections, MT boundaries in myelin-stained sections. The data are displayed on "unfolded" maps of the cortical surface created by the method of Van Essen and Maunsell (1980). Thin lines illustrate the layer 4 contours from which the maps were constructed. Thick lines show the boundaries of major sulci; dashed lines the fundi of major sulci. The solid black areas represent regions in which damage from the ibotenic acid extended through all 6 cortical layers. The dotted areas illustrate regions in which damage was restricted to a subset of laminae. $A$, The lesion that resulted from a small injection of ibotenic acid in experiment $m l$. It was largely restricted to MT, although a small area of damage was evident on the anterior bank of the STS. The anterior bank damage resulted from diffusion of the ibotenic acid from the injection site on the posterior bank. The oblique stripes indicate a region of uncertainty in the exact location of the MT boundary. $B$, The complete unilateral lesion of MT that resulted from 8 injections of ibotenic acid in experiment $m 2$. Only 2 myelin-stained sections yielded a hint of tissue that resembled MT. This locus is indicated by the arrow and small ellipse. This lesion included a portion of MST on the anterior bank of the STS, as well as the cntirc extent of MT. We also observed some damage in the lunate sulcus that resulted from backflow of the ibotenic acid up the guide tube tracks. $M T$, Middle temporal visual area; $S T S$, superior temporal sulcus; $S S$, sylvian sulcus; $L S$, lunate sulcus; $I P S$, intraparietal sulcus.
Figure $10 B$ illustrates the total extent of damaged cortex following the 8 injections of ibotenic acid in experiment $m 2$. An extensive region of cortex was destroyed on both banks of the STS, and a small region of damaged cortex appeared in the lunate sulcus (LS) as well. The damage in the lunate, which was largely restricted to layer 6 , resulted from backflow of the ibotenic acid up the guide tube tracks. The lesion on the posterior bank of the STS appears to have completely destroyed MT. We could find but one small region on 2 myelin-stained sections that may have been part of MT; this region is indicated by the arrow in Figure $10 B$.

The damaged cortex on the anterior bank of the STS in Figure $10 B$ includes a significant portion of the medial superior tem- poral area, or MST. MST extends beyond the damaged area in both posteromedial and anterolateral directions (Maunsell and Van Essen, 1983c), and the lesion of MST is therefore far from total. In the terminology of Desimone and Ungerleider (1986), the anterior bank lesion would probably include all of the middle temporal periphery (MTp), a substantial portion of MST, but very little of the floor of the superior temporal sulcus (FST).

\section{Discussion}

\section{$M T$ and motion processing}

The results of numerous physiological and anatomical studies suggest that MT is a major component of a cortical pathway that selectively analyzes visual motion information (see Maun- 
sell and Newsome, 1987). Recent behavioral studies have supported this hypothesis by showing that MT provides motion information for the guidance of eye movements (Newsome et al., 1985; Dursteler et al., 1987). The present results indicate that MT plays a role in the perception of motion as well. We have shown that small lesions of MT result in elevated psychophysical thresholds for a motion-sensitivity task, while thresholds for contrast sensitivity are affected little or not at all. Similar findings have been reported recently by Siegel and Andersen (1986). They trained monkeys to detect the onset of shearing motion and the onset of 3-dimensional structure in a structurefrom-motion task. MT lesions caused threshold elevations in both of these detection tasks, while leaving contrast-detection thresholds unimpaired.

Collectively, these studies provide significant insights into the functional role of MT in primate vision. First, they indicate that MT plays a role in a broad range of perceptual and behavioral functions, including direction discrimination, detection of shearing motion, detection of 3-dimensional structurc from motion, and guidance of eye movements to moving targets. These observations suggest that MT is best viewed as a general purpose motion processor whose outputs are used in a number of behavioral contexts. This does not, however, rule out the possibility that MT is more vital for certain motion-related tasks than for others. Motion information is employed by the visual system for far more purposes than have yet been examined with respect to MT function (see review by Nakayama, 1985). Future experiments may implicate MT more intimately in a restricted subset of these roles.

A second important consequence of these studies is that they confirm physiologically derived hypotheses concerning localization of function within extrastriate cortex. Zeki and his colleagues first showed that cortex in the STS contained an enriched concentration of directionally selective cells, and they proposed that this cortex was selectively involved in processing motion information (Dubner and Zeki, 1971; Zeki, 1974a, b). Recent studies, including the present one, have confirmed this hypothesis by demonstrating that MT lesions impair motion perception and eye movements to moving targets, but have no effect on contrast sensitivity or eye movements to stationary targets. It is likely that a more complete description of the selectivity of these lesions will emerge with continued study. For example, the physiology of MT neurons suggests that they may play a role in seeing motion in depth (Zeki, 1974b; Maunsell and Van Essen, 1983b), but should be irrelevant for seeing color (Zeki, 1978; Van Essen et al., 1981). It will be of interest to test both of these predictions with behavioral experiments.

\section{Recovery of function}

The rapidity with which monkeys recover from the effects of the lesions has been one of the more puzzling aspects of our experiments. In each of the major studies conducted to date (Newsome et al., 1985; Siegel and Andersen, 1986; Dursteler et al., 1987; and the present study), monkeys recover within several days from the effects of small ibotenic acid injections into MT. There is some evidence for a longer recovery period with a more complex perceptual task, such as the detection of 3-dimensional structure from motion (Siegel and Andersen, 1986), but the recovery period may only be lengthened in this case from days to weeks. The fact that recovery exists does not compromise our conclusions concerning the functional role of MT; our arguments are based on the selectivity of the lesion effects, not on their duration. Recovery of function demonstrates that a damaged hemisphere can generate alternative strategies for accomplishing certain tasks, but our results clearly show that lesions of MT disrupt the normal function of a pathway that selectively analyzes visual motion. Still, rapid recovery from such striking behavioral deficits begs for explanation.

We considered 3 possible explanations for the recovery (see Results): (1) The subtotal lesions caused by small injections of ibotenic acid did not eliminate the entire representation in MT of the stimulus aperture. (2) Plastic changes in topography or receptive-field size within MT might "fill in" the missing representation of the stimulus aperture. (3) Other visual areas operating in parallel with MT might assume the functions of lost MT cells. We attempted to differentiate among these possibilities by conducting electrophysiological recordings in hemisphere $m 2$ following recovery from an MT lesion. The recordings provided no evidence for extensive plastic changes in topography or receptive-field size, but they did indicate that some surviving receptive fields overlapped the stimulus aperture.

We therefore made a complete unilateral lesion of MT in this hemisphere with a series of 6 additional injections of ibotenic acid. This lesion caused a permanent elevation of motion thresholds, but the permanent impairment was small in comparison to the transient deficit that typically follows injections of ibotenic acid into MT (see Figs. 5-7, 9). In this case, therefore, it appears that a substantial portion of the recovery was mediated by other visual areas acting in parallel with MT. This result is not surprising in light of the complex anatomy of the motion pathway. MT sends projections to 2 higher cortical areas on this pathway, MST and VIP (Maunsell and Van Essen, 1983c; Ungerleider and Desimone, 1986), but each of these areas appears to receive inputs from lower visual areas, such as V2, as well (see Van Essen, 1985). If the additional inputs to MST and VIP provide motion information to these areas, then either area could plausibly assume some of the functions of damaged MT cells.

We cannot, however, rule out the possibility that intra-MT mechanisms mediate a portion of the recovery following small lesions. It is noteworthy that the second, more extensive, lesion of MT in experiment $m 2$ caused a full reinstatement of the transient deficit from which the monkey had already recovered (see Figs. 7,9). This observation would seem to imply that neurons within MT contributed to recovery from the initial lesion. However, an alternative interpretation is that an adjacent visual area, such as MST, mediated the initial recovery, and the second set of injections caused reinstatement of the original deficit because of diffusion of the ibotenic acid into MST. Indeed, histological reconstruction of the lesion in experiment $m 2$ (Fig. 10B) revealed a significant amount of damage in MST in addition to the complete unilatcral lesion of MT. This issuc must, unfortunately, remain unresolved until methods are available for creating even more precise lesions of individual visual areas.

It is natural to ask whether the rapid recovery observed in recent MT lesion studies implies that the truly unique functions of MT are yet to be discovered. This question arises from the view that each area of cortex has irreplaceable functions that should be reflected in robust, permanent deficits following destruction of that area. It seems likely, however, that many behavioral capabilities depend on neural activity that is distributed among several cortical areas. The potential for redundancy in 
such systems provides resistance to the deleterious effects of restricted lesions. From this perspective, the behavioral functions of a particular area may be best identified by techniques, such as those employed in the present study, that permit behavioral testing prior to the activation of compensatory mechanisms. Whether MT has unique functions in addition to the shared functions demonstrated thus far should become clear as a broader range of perceptual and behavioral tasks are examined in conjunction with MT lesions.

Note added in proof: Histological analysis has now confirmed that lesion $w 1$, like lesion $m l$ (see Fig. 10A), was largely restricted to MT, with minor damage occurring in the upper cortical layers of the anterior bank of the STS.

\section{References}

Albright, T. D. (1984) Direction and orientation selectivity of neurons in visual area MT of the macaque. J. Neurophysiol. 52: 1106-1130.

Allman, J. M., and J. H. Kaas (1971) A representation of the visual field in the caudal third of the middle temporal gyrus of the owl monkey (Aotus trivirgatus). Brain Res. 31: 85-105.

Anstis, S. M. (1980) The perception of apparent movement. Phil. Trans. R. Soc. Lond. [Biol.] 290: 153-168.

Baker, C. L., and O. J. Braddick (1985) Eccentricity dependent scaling of the limits for short-range apparent motion perception. Vision Res. 25: 803-812.

Blasdel, G. G., and D. Fitzpatrick (1984) Physiological organization of layer 4 in macaque striate cortex. J. Neurosci. 4: 880-895.

Braddick, O. (1974) A short-range process in apparent motion. Vision Res. 14: 519-527.

Braddick, O. J. (1980) Low level and high level processes in apparent motion. Phil. Trans. R. Soc. Lond. [Biol.] 290: 137-151.

Desimone, R, and L. G. Ungerleider (1986) Multiple visual areas in the caudal superior temporal sulcus of the macaque. J. Comp. Neurol. 248: 164-178

Dow, B. M. (1974) Functional classes of cells and their laminar distribution in monkey visual cortex. J. Neurophysiol. 37: 927-946.

Dubner, R., and S. M. Zeki (1971) Response properties and receptive fields of cells in an anatomically defined region of the superior temporal sulcus. Brain Res. 35: 528-532.

Dursteler, M. R., R. H. Wurtz, and W. T. Newsome (1987) Directional pursuit deficits following lesions of the foveal representation within the superior temporal sulcus of the macaque monkey. J. Neurophysiol. 57: 1262-1287.

Evarts, E. V. (1966) Methods for recording activity of individual neurons in moving animals. Methods Med. Res. 11: 241-250.

Evarts, E. V. (1968) A technique for recording activity of subcortical neurons in moving animals. Electroencephalogr. Clin. Neurophysiol. 24: 83-86.

Felleman, D. J., and D. C. Van Essen (1987) Receptive field properties of neurons in area $\mathrm{V} 3$ of macaque monkey extrastriate cortex. J. Neurosci. 4: 889-920.

Fuchs, A. F., and D. A. Robinson (1966) A method for measuring horizontal and vertical eye movement chronically in the monkey. J. Appl. Physiol. 21: 1068-1070.

Gallyas, F. (1979) Silver staining of myelin by means of physical development. Neurol. Res. 1: 203-209.

Judge, S. J., B. J. Richmond, and F. C. Chu (1980) Implantation of magnetic search coils for measurement of eye position: An improved method. Vision Res. 20: 535-538.

Levitt, H. (1971) Transformed up-down methods in psychoacoustics. J. Acoust. Soc. Am. 49: 467-477.

Livingstone, M. S., and D. H. Hubel (1984) Anatomy and physiology of a color system in the primate visual cortex. J. Neurosci. 4: 309356.

Maunsell, J. H. R., and W. T. Newsome (1987) Visual processing in monkey extrastriate cortex. Annu. Rev. Neurosci. 10: 363-401.

Maunsell, J. H. R., and D. C. Van Essen (1983a) Functional properties of neurons in the middle temporal visual area (MT) of the macaque monkey: I. Selectivity for stimulus direction, speed and orientation. J. Neurophysiol. 49: 1127-1147.

Maunsell, J. H. R., and D. C. Van Essen (1983b) Functional properties of neurons in the middle temporal visual area (MT) of the macaque monkey: II. Binocular interaction and sensitivity to binocular disparity. J. Neurophysiol. 49: 1148-1167.

Maunsell, J. H. R., and D. C. Van Essen (1983c) The connections of the middle temporal visual area (MT) and their relationship to a cortical hierarchy in the macaque monkey. J. Neurosci. 3: 2563-2586.

Merzenich, M. M., J. H. Kaas, J. Wall, R. J. Nelson, M. Sur, and D. Felleman (1983a) Topographic reorganization of somatosensory cortical areas 3B and 1 in adult monkeys following restricted deafferentation. Neuroscience 8: 33-55.

Merzenich, M. M., J. H. Kaas, J. T. Wall, M. Sur, and R. J. Nelson (1983b) Progression of change following median nerve section in the cortical representation of the hand in areas $3 \mathrm{~b}$ and 1 in adult owl and squirrel monkeys. Neuroscience 10: 639-665.

Merzenich, M. M., R. J. Nelson, M. P. Stryker, M. S. Cynader, A. Schoppmann, and J. M. Zook (1984) Somatosensory cortical map changes following digit amputation in adult monkeys. J. Comp. Neurol. 224: 591-605.

Michael, C. R. (1985) Laminar segregation of color cells in the monkey striate cortex. Vision Res. 25: 415-423.

Morgan, M. J., and R. Ward (1980) Conditions for motion flow in dynamic visual noise. Vision Res. 20: 431-435.

Nakayama, K. (1985) Biological image motion processing: A review. Vision Res. 25: 625-660.

Nakayama, K., and C. W. Tyler (1981) Psychophysical isolation of movement sensitivity by removal of familiar position cues. Vision Res. 21: 427-433.

Newsome, W. T., and E. B. Paré (1986) MT lesions impair discrimination of direction in a stochastic motion display. Soc. Neurosci. Abstr. 12: 1183 .

Newsome, W. T., R. H. Wurtz, M. R. Dursteler, and A. Mikami (1985) Deficits in visual motion processing following ibotenic acid lesions of the middle temporal visual area of the macaque monkey. J. Neurosci. 5: $825-840$.

Olney, J. W. (1983) Excitotoxins: An overview. In Excitotoxins, K. Fuxe, P. Roberts, and R. Schwartz, eds., pp. 82-95, Macmillan, London.

Robinson, D. A. (1963) A method of measuring eye movement using a scleral search coil in a magnetic field. IEEE Trans. Biomed. Eng. 10: $137-145$.

Siegel, R. M., and R. A. Andersen (1986) Motion perceptual deficits following ibotenic acid lesions of the middle temporal area (MT) in the behaving rhesus monkey. Soc. Neurosci. Abstr. 12: 1183.

Tanaka, K., H. Hikosaka, H. Saito, Y. Yukie, Y. Fukada, and E. Iwai (1986) Analysis of local and wide field movements in the superior temporal visual areas of the macaque monkey. J. Neurosci. 6: 134144.

Ungerleider, L. G., and R. Desimone (1980) Cortical connections of visual area MT in the macaque. J. Comp. Neurol. 248: 190-222.

Van Essen, D. C. (1985) Functional organization of primate visual cortex. In Cerebral Cortex, A. Peters and E. G. Jones, eds., pp. 259329, Plenum, New York.

Van Essen, D. C., and J. H. R. Maunsell (1980) Two-dimensional maps of the cercbral cortex. J. Comp. Neurol. 191: 255-281.

Van Essen, D. C., J. H. R. Maunsell, and J. L. Bixby (1981) The middle temporal visual area in the macaque: Myeloarchitecture, connections, functional properties and topographic representation. J. Comp. Neurol. 199: 293-326.

Williams, D. W., and R. Sekuler (1984) Coherent global motion percepts from stochastic local motions. Vision Res. 24: 55-62.

Zeki, S. M. (1974a) Functional organization of a visual area in the posterior bank of the superior temporal sulcus of the rhesus monkey. J. Physiol. (Lond.) 236: 549-573.

Zeki, S. M. (1974b) Cells responding to changing image size and disparity in the cortex of the rhesus monkey. J. Physiol. (Lond.) 242. 827-841.

Zeki, S. M. (1978) Uniformity and diversity of structure and function in rhesus monkey prestriate visual cortex. J. Physiol. (Lond.) 277: $273-290$ 